\title{
Insight into the Role of Instantons and their Zero Modes from Lattice QCD
}

\author{
J.W. Negele ${ }^{a *}$ \\ ${ }^{a}$ Center for Theoretical Physics \\ Laboratory for Nuclear Science and Department of Physics \\ Massachusetts Institute of Technology, Cambridge MA 02139
}

Evidence from lattice QCD calculations is presented showing that instantons and their associated zero modes play a major role in the physics of light hadrons and the propagation of light quarks in the QCD vacuum.

\section{Introduction and Motivation}

During the quarter of a century I have known Koichi Yazaki as a colleague, collaborator and friend, we have shared an abiding interest in many body physics - understanding how strongly interacting systems develop their rich and complex properties from their fundamental underlying interactions. Hence, it is a special pleasure and privilege to be able to take part in this symposium being held in his honor and to talk about insight my collaborators and I have gotten from lattice QCD into the role of instantons and their associated zero modes in the physics of hadrons.

One of the great advantages of the path integral formulation of quantum mechanics and field theory is the possibility of identifying non-perturbatively the stationary configurations that dominate the action and thereby identify and understand the essential physics of complex systems with many degrees of freedom. Thus, the discovery of instantons in 1975 [1] gave rise to great excitement and optimism that they were the key to understanding QCD. Indeed, in contrast to other many body systems in which the quanta exchanged between interacting fermions can be subsumed into a potential, it appeared that QCD was fundamentally different, with topological excitations of the gluon field dominating the physics and being responsible for a host of novel and important effects including the $\theta$ vacuum, the axial anomaly, fermion zero modes, the mass of the $\eta^{\prime}$, and the chiral condensate. However, despite nearly a quarter of a century of theoretical effort, it has not been possible to proceed analytically beyond the dilute instanton gas approximation [2]. In the intervening years, the instanton liquid model [3/5] provided a successful phenomenology and qualitative physical understanding, but a quantitative exploration of the role of instantons in nonperturbative QCD has had to wait until lattice QCD became sufficiently sophisticated and sufficient resources could be devoted to the study of instanton physics.

\footnotetext{
${ }^{*}$ This work is supported in part by funds provided by the US Department of Energy (DOE) under cooperative research agreement \#DF-FC02-94ER40818.
} 
Our basic strategy will be to reverse the usual analytical process of calculating stationary configurations and approximately summing the fluctuations around them. Rather, we will use Monte Carlo sampling of the path integral for QCD on a lattice to identify typical paths contributing to the action and then work backwards to identify the smooth classical solutions about which these paths are fluctuating. The physical picture that arises corresponds closely to the physical arguments and instanton models of Shuryak and Diakonov 3 -5 in which the zero modes associated with instantons produce localized quark states, and quark propagation proceeds primarily by hopping between these states.

\subsection{Aspects of continuum instanton physics}

To put lattice investigations in context, it is useful to recall relevant aspects of continuum instanton physics. Working in Euclidean time, we evaluate a path integral of the form $\int \mathcal{D}[A] e^{\int d^{4} x S[A]}$. Hence, as in statistical mechanics, the weight of a configuration depends not only on its energy, but also on its entropy - the number of ways it can be realized. In addition, tunneling solutions arise as periodic classical solutions in an inverted potential. Hence, we may expect BPST instantons [1] which connect degenerate minima of differing winding number with the self-dual gauge potential $A_{\mu}^{a}(x)=\frac{2 \eta_{a \mu \nu} x_{\nu}}{x^{2}+\rho^{2}}$ having scale-invariant action $S=\frac{1}{4} \int d^{4} x F_{\mu \nu}^{a} F_{\mu \nu}^{a}=8 \pi^{2} g^{-2} \equiv S_{0}$ to have a significant presence in the vacuum due to the high entropy associated with translation, size, and color orientation. The action and topological charge density are localized around the center as $\pm F_{\mu \nu}^{a} \tilde{F}_{\mu \nu}^{a}=F_{\mu \nu}^{a} F_{\mu \nu}^{a}=\frac{192 \rho^{4}}{\left(x^{2}+\rho^{2}\right)^{4}}$. The $d n_{I} \sim\left(\frac{8 \pi^{2}}{g^{2}}\right)^{2 N_{c}} \frac{d \rho}{\rho^{5}} d^{4} x^{(I)} \exp \left\{-\frac{8 \pi^{2}}{g^{2}\left(\Lambda^{-1}\right)}\right\}(\Lambda \rho)^{\frac{11 N_{c}}{3}}$, where the prefactor and the running of the coupling constant in the last factor produce a distribution of instantons $\sim \rho^{6}$ for $\mathrm{SU}(3)$. Physically, we expect this distribution to be cut off at large $\rho$ by interactions between instantons and by fluctuations when the amplitude of a sufficiently large instanton becomes small relative to quantum fluctuations. It is the difficulty in treating these infrared effects that has stymied analytic progress.

From the axial anomaly, $\partial_{\mu} \sum_{f} \bar{\psi} \gamma_{\mu} \gamma_{5} \psi=2 m \sum_{f} \bar{\psi} \gamma_{5} \psi+\frac{N_{f}}{16 \pi^{2}} F_{\mu \nu}^{a} \tilde{F}_{\mu \nu}^{a}$, the topological charge satisfies the index theorem and, for periodic systems, may be expressed in terms of fermion eigenfunctions $Q=\frac{g^{2}}{32 \pi^{2}} \int F \tilde{F}=n_{L}-n_{R}=m \sum_{\lambda} \frac{\int \psi_{\lambda}^{\dagger}(x) \gamma_{5} \psi_{\lambda}(x)}{m+i \lambda}$, where $n_{L}$ and $n_{R}$ denote the number of fermion zero modes. For an isolated instanton, the zero mode is $\psi_{0}(x)=\frac{\rho \gamma \cdot \widehat{x}\left(1+\gamma_{5}\right)}{2 \pi\left(x^{2}+\rho^{2}\right)^{3 / 2}} \phi$. In the limit of light quarks, the Greens function for $\mathrm{N}_{f}$ quarks reduces to the product of zero modes $\prod_{f} \operatorname{det}[\not D+m] \bar{\psi}_{f}(x) \psi_{f}(y) \underset{m \rightarrow 0}{\longrightarrow} \prod_{f} \psi_{0}(x) \psi_{0}^{\dagger}(y)$ and gives rise to the 't Hooft interaction. Thus, light quarks propagate by zero modes which in turn arise from instantons. Based on large $\mathrm{N}$ arguments, the Veneziano-Witten formula [6,7] relates the $\eta^{\prime}$ mass to the topological susceptibility in the pure gluon sector $\chi \equiv \int \frac{d^{4} x}{V}\langle Q(x) Q(0)\rangle=\frac{f_{\pi}^{2}}{2 N_{f}}\left(m_{\eta}^{2}+m_{\eta^{\prime}}^{2}-2 m_{K}^{2}\right)$ yielding the expectation that $\chi=(180 \mathrm{MeV})^{4}$.

The instanton liquid model [3] provides an economical phenomenology of instanton mediated quark propagation in the QCD vacuum. The integral over all gluon fields that one evaluates in lattice QCD using an ensemble of configurations sampling the action is replaced by an ensemble of instantons and antiinstantons of size $\rho \sim 1 / 3 \mathrm{fm}$ and density $n \sim 1 \mathrm{fm}^{4}$ randomly distributed in space and color orientation, where the values of $\rho$ and $n$ are determined from the physical gluon and chiral condensates. One may think of the 't Hooft interaction as a vertex that absorbs left-handed particles of each flavor and creates corresponding right-handed particles, and vice versa for antiinstantons. Mesons 
then propagate in the QCD vacuum by the hopping of quark-antiquark pairs between these vertices, and the qualitative features of the channel dependence arises naturally with the pion channel being strongly attractive, the scalar channel repulsive, and the interaction in the rho channel very weak. The chiral condensate arises naturally in this picture by the fact that the zero modes for isolated instantons mix in the instanton liquid giving rise to a finite density of states at low virtuality.

\subsection{Lattice QCD}

A QCD observable is evaluated by defining quark and gluon variables on the sites and links of a space-time lattice, writing a Euclidean path integral of the generic form 8 $\left\langle T e^{-B \widehat{H}} \hat{\bar{\psi}} \widehat{\bar{\psi}} \widehat{\psi} \widehat{\psi}\right\rangle=Z^{-1} \int \mathcal{D}(U) \mathcal{D}(\bar{\psi} \psi) e^{-\bar{\psi} M(U) \psi-S(U)} \bar{\psi} \bar{\psi} \psi \psi$

$=Z^{-1} \int \mathcal{D}(U) e^{\ln \operatorname{det} M(U)-S(U)} M^{-1}(U) M^{-1}(U)$ and evaluating the final integral over gluon link variables $U$ using the Monte Carlo method. The link variable is $U=e^{i a g A_{\mu}(x)}$, the Wilson gluon action is $S(U)=\frac{2 n}{g^{2}} \sum_{\square}\left(1-\frac{1}{N} \operatorname{Re} \operatorname{Tr} U_{\square}\right)$ where $U_{\square}$ denotes the product of link variables around a single plaquette, and $M(U)$ denotes the discrete Wilson approximation to the inverse propagator $M(U) \rightarrow m+\not \partial+i g \not$.

Vacuum correlation functions for space-like separated hadron currents calculated in lattice QCD display the qualitative behavior expected from the 't Hooft interaction and agree semi-quantitatively with the instanton liquid model. As emphasized in ref 9], correlation functions of the form $R(x) \equiv\left\langle 0\left|T J_{\mu}(x) J_{\mu}(0)\right| 0\right\rangle$ characterize the spatial and channel dependence of the interaction between quarks and antiquarks and thus supplement hadron bound state properties like phase shifts supplement deuteron properties in characterizing the nuclear interaction. The ratio $R(x) / R_{0}(x)$ of the interacting to free correlator has been calculated in quenched QCD for the following meson and baryon currents [10], $J=\bar{u} \gamma_{\mu} d, \bar{u} \gamma_{\mu} \gamma_{5} d, \bar{u} \gamma_{5} d, \bar{u} d, \epsilon_{a b c}\left[c^{a} C \gamma_{\mu} u^{b}\right] \gamma_{\mu} \gamma_{5} d^{c}$, and $\epsilon_{a b c}\left[u^{a} C \gamma_{\mu} u^{b}\right] u^{c}$. Results are consistent with dispersion analysis of $\mathrm{e}^{+}-\mathrm{e}^{-}$and other data in relevant channels, and agree in detail with the channel dependence expected from the 't Hooft interaction and the instanton liquid model. Note for subsequent reference, that all correlation functions may be decomposed into a continuum contribution concentrated near the origin and a resonance contribution arising from the lowest bound state or resonance which dominates in the region of $1 \mathrm{fm}$. Quenched calculations at $\beta=6.2$ [11 corroborate the original $\beta=5.7$ results.

\section{Extraction of the instanton content of lattice gluon configurations}

\subsection{Identifying instantons by cooling}

The Feynman path integral for a quantum mechanical problem with degenerate minima is dominated by paths that fluctuate around stationary solutions to the classical Euclidean action connecting these minima. In the case of the double well potential, a typical Feynman path is composed of segments fluctuating around the left and right minima joined by segments crossing the barrier. If one had such a trajectory as an initial condition,

one could find the nearest stationary solution to the classical action numerically by using an iterative local relaxation algorithm. In this method, which has come to be known as cooling, one sequentially minimizes the action locally as a function of the coordinate on each time slice and iteratively approaches a stationary solution. In the case of the double 
well, the trajectory approaches straight lines in the two minima joined by kinks and antikinks crossing the barrier and the structure of the trajectory can be characterized by the number and positions of the kinks and anti-kinks.

In QCD, the corresponding classical stationary solutions to the Euclidean action for the gauge field connecting degenerate minima of the vacuum are instantons, and the analogous cooling technique 12,13 reveals the instantons corresponding to each gauge field configuration.

The results of using 25 cooling steps as a filter to extract the instanton content of a typical gluon configuration are shown in Fig. 1 of Ref. [14] using the Wilson action on a $16^{3} \times 24$ lattice at $6 / g^{2}=5.7$. As one can see, there is no recognizable structure before cooling. Large, short wavelength fluctuations of the order of the lattice spacing dominate both the action and topological charge density. After 25 cooling steps, three instantons and two anti-instantons can be identified clearly. The action density peaks are completely correlated in position and shape with the topological charge density peaks for instantons and with the topological charge density valleys for anti-instantons. Note that both the action and topological charge densities are reduced by more than two orders of magnitude so that the fluctuations removed by cooling are several orders of magnitude larger than the topological excitations that are retained.

\subsection{The QCD Vacuum}

Several extensive studies of the instanton content of the $\mathrm{SU}(3)$ vacuum have been performed recently and are reviewed in ref [15]. The results consistently indicate that the average size of an instanton when extrapolated to the uncooled vacuum is $0.39 \pm .05$ $\mathrm{fm}$, in agreement with the liquid instanton model, and that the topological susceptibility is $\chi \sim 180 \mathrm{MeV}$, in agreement with the Veneziano-Witten formula.

\subsection{Comparison of results with all gluons and with only instantons}

One dramatic indication of the role of instantons in light hadrons is to compare observables calculated using all gluon contributions with those obtained using only the instantons remaining after cooling. Note that there are truly dramatic differences in the gluon content before and after cooling. Not only has the action density decreased by two orders of magnitude, but also the string tension has decreased to $27 \%$ of its original value and the Coulombic and magnetic hyperfine components of the quark-quark potential are essentially zero. Hence, for example, the energies and wave functions of charmed and $B$ mesons would be drastically changed.

As shown in Fig. 1, however, when the coupling constant, or equivalently, the lattice spacing, and quark mass are set by the nucleon and pion masses in the usual way, the properties of the rho meson are virtually unchanged. The vacuum correlation function in the rho (vector) channel and the spatial distribution of the quarks in the rho ground state, given by the ground state density-density correlation function [16] $\left\langle\rho\left|\bar{q} \gamma_{0} q(x) \bar{q} \gamma_{0} q(0)\right| \rho\right\rangle$, are statistically indistinguishable before and after cooling. Also, as shown in Ref. [14], the rho mass is unchanged within its $10 \%$ statistical error. In addition, the pseudoscalar, nucleon, and delta vacuum correlation functions and nucleon and pion density-density correlation functions are also qualitatively unchanged after cooling, except for the removal of the small Coulomb induced cusp at the origin of the pion. Similarly, the axial charge matrix

elements specifying the spin content of the nucleon, $\left\langle\vec{P} \vec{S}\left|\left(\bar{q} \gamma^{i} i \gamma_{5} q\right)\right| \vec{P} \vec{S}\right\rangle=2 S^{i} \Delta q$, are 

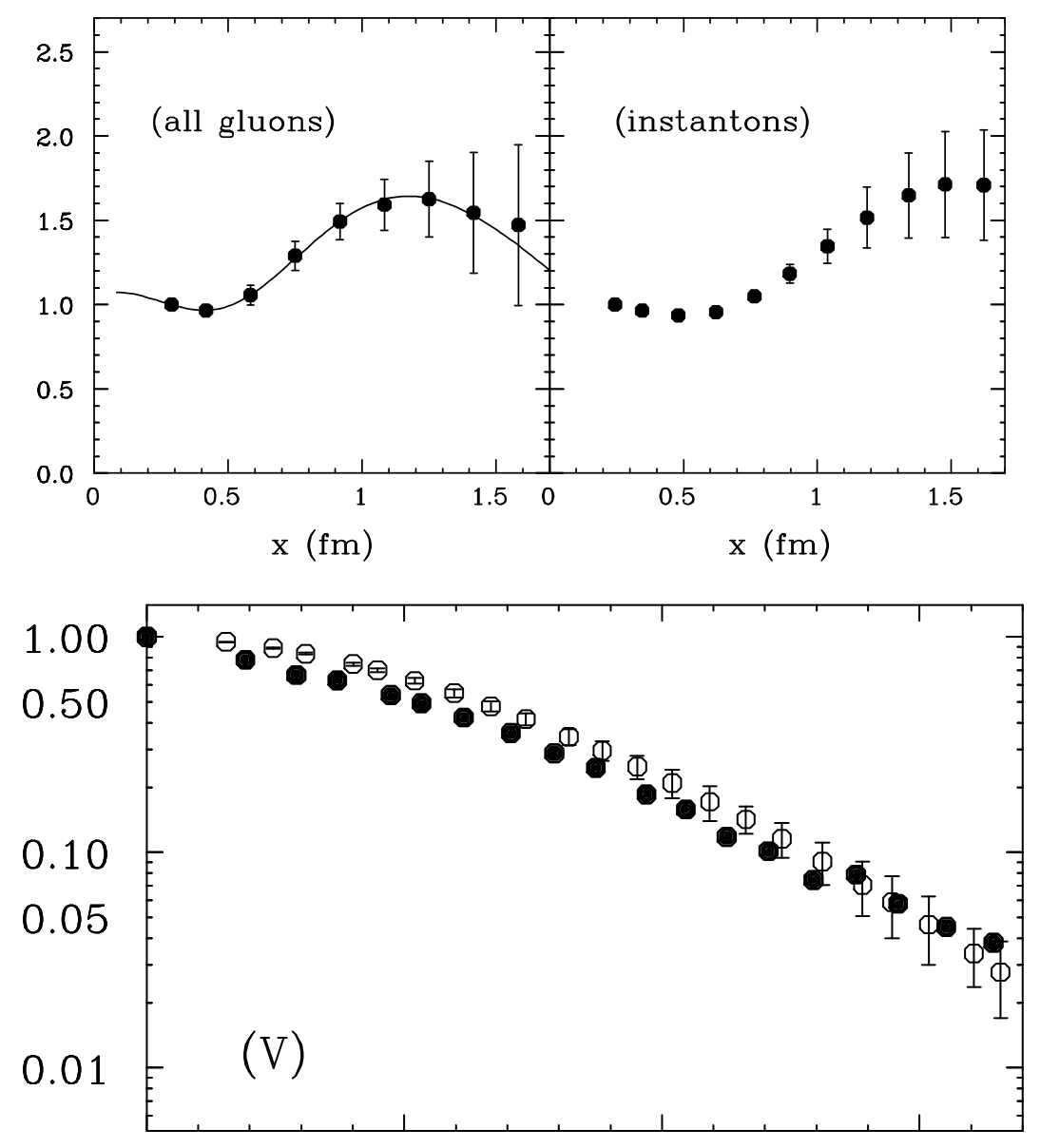

Figure 1. Comparison of rho observables calculated with all gluon configurations and only instantons. The upper left-hand plot shows the vacuum correlator in the rho channel calculated with all gluons and the upper right-hand plot shows the analogous result with only instantons. The lower plot shows the ground state density-density correlation function for the rho with all gluons (solid circles) and with only instantons (open circles).

quite similar when calculated with all gluons and only instantons [17].

\section{Quark Zero Modes and their contributions to light hadrons}

\subsection{Eigenmodes of the Dirac operator}

In the continuum limit, the Dirac operator for Wilson fermions, $D \psi_{x}=\psi_{x}-\kappa \sum_{\mu}\left[\left(r-\gamma_{\mu}\right) u_{x, \mu} \psi_{x+\mu}+\left(r+\gamma_{\mu}\right) u_{x-\mu, \mu}^{\dagger} \psi_{x-\mu}\right]$, approaches the familiar continuum result $\frac{1}{m}[m+i(\not p+g \not A)] \psi$.

In the free case, the continuum spectrum is $\frac{1}{m}[m+i|\vec{p}|]$ and the Wilson lattice operator approximates this spectrum in the physical regime and pushes the unphysical fermion modes to very large (real) masses. In the presence of an instanton of size $\rho$ at $x=0$, it is shown in Ref. [18 that the lattice operator produces a mode with a real eigenvalue which approaches the continuum result $\psi_{0}(x)_{s, \alpha}=u_{s, \alpha} \frac{\sqrt{2}}{\pi} \frac{\rho}{\left(x^{2}+\rho^{2}\right)^{3 / 2}}$ and whose mixing with other 

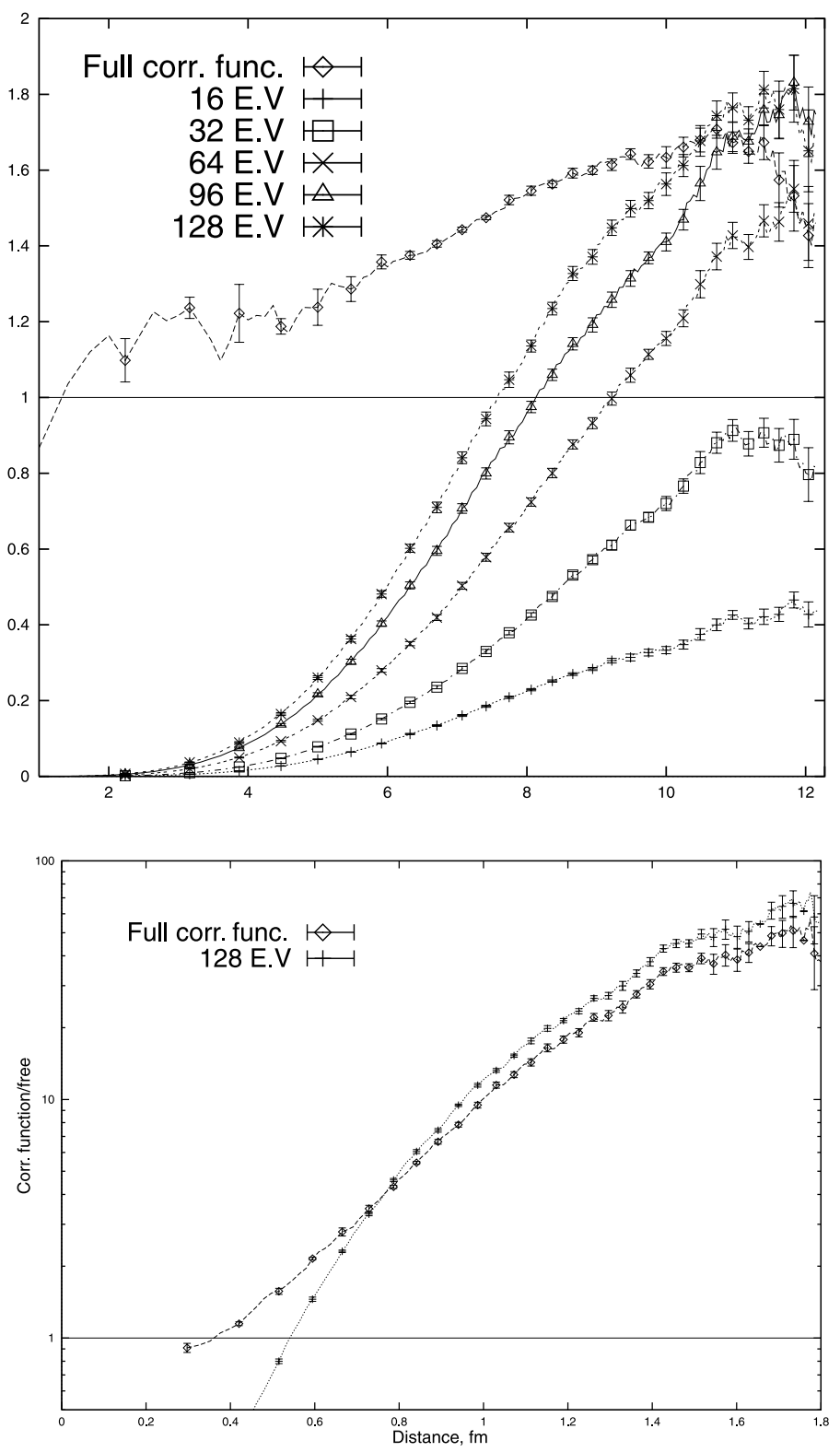

Figure 2. Contributions of low Dirac eigenmodes to the vector (upper graph) and pseudoscalar (lower graph) vacuum correlation functions. The upper graph shows the contributions of 16, 32, 64, 96, and 128 eigenmodes compared with the full correlation function for an unquenched configuration and the lower graph compares 128 eigenmodes with the full correlation function for a quenched configuration.

modes goes to zero as the lattice volume goes to infinity. In addition, instanton-antiinstanton pairs that interact sufficiently form complex conjugate pairs of eigenvalues that move slightly off the real axis. Thus, by observing the Dirac spectrum for a lattice gluon configuration containing a collection of instantons and anti-instantons, it is possible to identify zero modes directly in the spectrum. 
Ref. [18] shows the lowest 64 complex eigenvalues of the Dirac operator on a $16^{4}$ unquenched gluon configuration for $6 / g^{2}=5.5$ and $\kappa=0.16$, both before and after cooling (where 100 relaxation steps with a parallel algorithm are comparable to 25 cooling steps). The cooled plot has just the structure we expect with a number of isolated instantons with modes on the real axis and pairs of interacting instantons slightly off the real axis. However, even though the uncooled case also contains fluctuations several orders of magnitude larger than the instantons, it shows the same structure of isolated instantons and interacting pairs, clearly revealing the zero modes in the original, uncooled vacuum.

\subsection{Zero mode expansion}

The Wilson-Dirac operator has the property that $D=\gamma_{5} D^{\dagger} \gamma_{5}$, which implies that $\left\langle\psi_{j}\left|\gamma_{5}\right| \psi_{i}\right\rangle=0$ unless $\lambda_{i}=\lambda_{j}^{*}$ and we may write the spectral representation of the prop-

agator $\left\langle x\left|D^{-1}\right| y\right\rangle=\sum_{i} \frac{\left\langle x \mid \psi_{i}\right\rangle\left\langle\psi_{\bar{i}}\left|\gamma_{5}\right| y\right\rangle}{\left\langle\psi_{\bar{i}}\left|\gamma_{5}\right| \psi_{i}\right\rangle \lambda_{i}}$ where $\lambda_{i}=\lambda_{i}^{*}$. A clear indication of the role of zero modes in light hadron observables is the degree to which truncation of the expansion to the zero mode zone reproduces the result with the complete propagator.

Fig. 2 shows the result of truncating the vacuum correlation functions for the vector and pseudoscalar channels to include only low eigenmodes 18. On a $16^{4}$ lattice, the full propagator contains 786,432 modes. The top plot of Fig. 2 shows the result of including the lowest $16,32,64,96$, and finally 128 modes. Note that the first 64 modes reproduce most of the strength in the rho resonance peak, and by the time we include the first 128 modes, all the strength is accounted for. Similarly, the lower plot in Fig. 2 shows that the lowest 128 modes also account for the analogous pion contribution to the pseudoscalar vacuum correlation function. Similarly, most of the strength of the disconnected graph contribution to the $\eta^{\prime}$ correlation function, which should be particularly sensitive to instantons, is already provided by the lowest 32 eigenmodes, and the fermionic definition of the topological charge is nearly saturated by the lowest 8 modes [19].

\subsection{Localization}

Finally, it is interesting to ask whether the lattice zero mode eigenfunctions are localized on instantons. This was studied by plotting the quark density distribution for individual eigenmodes in the $x$ - $z$ plane for all values of $y$ and $t$, and comparing with analogous plots of the action density. As expected, for a cooled configuration the eigenmodes correspond to linear combinations of localized zero modes at each of the instantons. What is truly remarkable, however, is that the eigenfunctions of the uncooled configurations also exhibit localized peaks at locations at which instantons are identified by cooling. Thus, in spite of the fluctuations several orders of magnitude larger than the instanton fields themselves, the light quarks essentially average out these fluctuations and produce localized peaks at the topological excitations. When one analyzes a number of eigenfunctions, one finds that all the instantons remaining after cooling correspond to localized quark fermion peaks in some eigenfunctions. However, some fermion peaks are present for the initial gluon configurations that do not correspond to instantons that survive cooling. These presumably correspond to instanton-anti-instanton pairs that were annihilated during cooling. 


\section{Conclusion}

Altogether, the lattice calculations reported here provide strong evidence that instantons play a dominant role in quark propagation in the vacuum and in light hadron structure. We have shown that the instanton content of gluon configurations can be extracted by cooling, and that the instanton size is consistent with the instanton liquid model and the topological susceptibility agrees with the Veneziano-Witten formula. We obtain striking agreement between vacuum correlation functions, ground state density-density correlation functions, and masses calculated with all gluons and with only instantons. Zero modes associated with instantons are clearly evident in the Dirac spectrum, and account for the $\rho, \pi$, and $\eta \prime$ contributions to vacuum correlation functions. Finally, we have observed directly quark localization at instantons in uncooled configurations.

\section{Acknowledgments}

It is a pleasure to acknowledge the essential role of Richard Brower, Ming Chu, Dmitri Dolgov, Jeff Grandy, Suzhou Huang, Taras Ivananko, Kostas Orginos, and Andrew Pochinsky who collaborated in various aspects of this work. We are also grateful for the donation by Sun Microsystems of the 24 Gflops E5000 SMP cluster on which the most recent calculations were performed and the computer resources provided by NERSC with which this work was begun.

\section{REFERENCES}

1. A.A. Belavin, A.M. Polyakov, A.P. Schwartz, and Y.S. Tyupkin, Phys. Lett. B 59, 85 (1975).

2. C.G. Callan, R. Dashen, and D. J. Gross, Phys. Rev. D 17, 2717 (1978).

3. E.V. Shuryak Nucl. Phys. B 198, 83 (1982).

4. D.I. Diakonov and V.Y. Petrov, Nucl. Phys. B 245, 259 (1984); 272, 457 (1986).

5. T. Schäffer and E.V. Shuryak, Rev. Mod. Phys. 70, 323 (1998), and references therein.

6. E. Witten, Nucl. Phys. B 156, 269 (1979).

7. G. Veneziano, Nucl. Phys. B 159, 213 (1979).

8. J. W. Negele, Varenna lectures, hep-lat/9804017.

9. E.V. Shuryak, Rev. Mod. Phys. 65, 1 (1993).

10. M.-C. Chu, J.M. Grandy, S. Huang, and J.W. Negele, Phys. Rev. D 48, 3340 (1993).

11. S.J. Hands, P.W. Stephenson, A. McKerrell, Phys. Rev. D 51, 6394 (1995).

12. B. Berg, Phys. Lett. B 104, 475 (1981).

13. M. Teper, Nucl. Phys. B (Proc.Suppl.) 20, 159 (1991).

14. M.-C. Chu, J.M. Grandy, S. Huang, and J.W. Negele, Phys. Rev. D 49, 6039 (1994).

15. J. W. Negele, hep-lat/9810053.

16. M.-C. Chu, M.Lissia, and J.W. Negele, Nucl. Phys. B 360, 31 (1991); M. Lissia, M.-C. Chu, J.W. Negele, and J.M. Grandy, Nucl. Phys. A 555, 272 (1993).

17. D. Dolgov, R.C. Brower, J.W. Negele, and A. Pochinhsky, hep-lat/9809132.

18. T.L. Ivanenko and J.W. Negele, Nucl. Phys. B (Proc.Suppl.) 63, 504 (1998); T.L. Ivanenko, MIT Ph.D. thesis, 1997; J.W. Negele, hep-lat/9804017.

19. L. Venkataraman and G. Kilcup, hep-lat/9711006. 